Leonid Polterovich

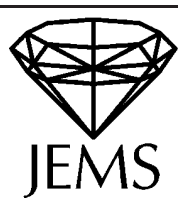

\title{
Hamiltonian loops from the ergodic point of view
}

Received July 7, 1998 / final version received September 14, 1998

\begin{abstract}
Let $G$ be the group of Hamiltonian diffeomorphisms of a closed symplectic manifold $Y$. A loop $h: S^{1} \rightarrow G$ is called strictly ergodic if for some irrational number $\alpha$ the associated skew product map $T: S^{1} \times Y \rightarrow S^{1} \times Y$ defined by $T(t, y)=(t+\alpha, h(t) y)$ is strictly ergodic. In the present paper we address the following question. Which elements of the fundamental group of $G$ can be represented by strictly ergodic loops? We prove existence of contractible strictly ergodic loops for a wide class of symplectic manifolds (for instance for simply connected ones). Further, we find a restriction on the homotopy classes of smooth strictly ergodic loops in the framework of Hofer's bi-invariant geometry on $G$. Namely, we prove that their asymptotic Hofer's norm must vanish. This result provides a link between ergodic theory and symplectic topology.
\end{abstract}

\section{Introduction and results}

\subsection{Hamiltonian loops as dynamical objects}

Let $(Y, \Omega)$ be a closed symplectic manifold, and let $G$ be its group of Hamiltonian diffeomorphisms. ${ }^{1}$ Given an irrational number $\alpha$ and a smooth loop $h: S^{1} \rightarrow G$, one can define a skew product map $T_{h, \alpha}: S^{1} \times Y \rightarrow S^{1} \times Y$ by $T_{h, \alpha}(t, y)=(t+\alpha, h(t) y)$. The purpose of the present paper is to relate geometry and topology of Hamiltonian loops with dynamics of associated skew products.

The definition above is a particular case of a much more general notion of a skew product (see [CFS, p. 231]) which has been intensively studied during several decades. There are at least two important reasons for the interest to this notion. First, it serves as a foundation for mathematical models of random dynamics (see [Ki] for a survey). Second, it provides non-trivial examples of systems with interesting dynamical properties (see discussion in 1.2 below).

L. Polterovich: School of Mathematical Sciences, Tel Aviv University, 69978 Tel Aviv, Israel, e-mail: polterov@math.tau.ac.il

Supported by the United States - Israel Binational Science Foundation grant 94-00302

Mathematics Subject Classification (1991): 58Dxx, 58F05, 28D05

1 Recall that $G$ consists of all symplectomorphisms of $(Y, \Omega)$ which can be included into a time-dependent Hamiltonian flow on $Y$. 
The dynamical property we consider is the strict ergodicity. Recall that a homeomorphism $T$ of a compact topological space $X$ is called strictly ergodic if it has precisely one invariant Borel probability measure, say $\mu$, and moreover this measure is positive on non-empty open subsets. Strictly ergodic homeomorphisms are ergodic, and have a number of additional remarkable features. We mention one of them which plays a crucial role below. Namely, given such a $T$ and an arbitrary continuous function $F$ on $X$, the time averages $\frac{1}{N} \sum_{i=0}^{N-1} F\left(T^{i} x\right)$ converge uniformly to the space average $\int_{X} F d \mu$, and in particular converge for all $x \in X$. Note that for general ergodic transformations such a convergence takes place only for almost all $x$. The contrast between "all" and "almost all" becomes especially transparent when one notices that there are pure topological obstructions to the strict ergodicity. For instance, the 2-sphere admits no strictly ergodic homeomorphisms. Indeed the Lefschetz theorem implies that every homeomorphism of $S^{2}$ has either a fixed point, or a periodic orbit of period 2 and we see that the invariant measure which is concentrated on such an orbit contradicts the definition of strict ergodicity. In 1.3 below we describe a more sophisticated obstruction to the strict ergodicity which comes from symplectic topology.

We say that a loop $h: S^{1} \rightarrow G$ is strictly ergodic if for some $\alpha$ the corresponding skew product map $T_{h, \alpha}$ is strictly ergodic. ${ }^{2}$ With this language our central question can be formulated as follows.

Question 1.1.A. Which homotopy classes $S^{1} \rightarrow G$ can be represented by strictly ergodic loops?

Here is an example where one gets a complete answer to this question. Let $Y$ be the blow up of the complex projective plane $\mathbf{C P}^{2}$ at one point. Choose a Kähler symplectic structure $\Omega$ on $Y$ which integrates to 1 over a general line and to $\frac{1}{3}$ over the exceptional divisor. The periods of the symplectic form are chosen in such a way that its cohomology class is a multiple of the first Chern class of $Y$. One can easily see that $(Y, \Omega)$ admits an effective Hamiltonian action of the unitary group $U(2)$, in other words there exists a monomorphism $i: U(2) \rightarrow G$. The fundamental group of $U(2)$ equals $\mathbf{Z}$. It was proved recently by Abreu and McDuff [AM] that the inclusion $\pi_{1}(U(2)) \rightarrow \pi_{1}(G)$ is an isomorphism, and thus $\pi_{1}(G)=\mathbf{Z}$. As far as we know this is the simplest example of a symplectic manifold with $\pi_{1}(G)=\mathbf{Z}$.

Theorem 1.1.B. In this situation, the trivial class $0 \in \mathbf{Z}$ is the only one which can be represented by a strictly ergodic loop.

\footnotetext{
2 Note that each $T_{h, \alpha}$ preserves the canonical measure on $S^{1} \times Y$ induced by the symplectic form. Thus in our setting the strict ergodicity means that this measure is the unique (up to a factor) invariant measure.
} 
The proof (see 1.3 below) is based on two general results on existence and non-existence of strictly ergodic loops.

\subsection{An existence result}

Assume in addition that the group $G$ of all Hamiltonian diffeomorphisms of a closed symplectic manifold $(Y, \Omega)$ is $C^{\infty}$-closed in $\operatorname{Diff}(Y)$.

Theorem 1.2.A. Under this assumption there exists a contractible smooth strictly ergodic Hamiltonian loop.

Remark. The assumption above holds for a wide class of symplectic manifolds, for instance when $H^{1}(Y, \mathbf{R})=0$, or when the cohomology class of the symplectic form is rational. (And thus it holds for the blow up of $\mathbf{C P}^{2}$ considered in 1.1 above). However it is still unclear whether it is valid for all closed symplectic manifolds. This long standing problem is known as the Flux conjecture, and we refer the reader to [LMP] for more discussion and recent results. On the other hand it sounds likely that in our situation this assumption plays a technical role only and can be removed, but I have not checked the details (see the footnote in 5.3 below; I am thankful to D. McDuff for illuminating discussions on this issue).

Constructions of ergodic and strictly ergodic skew products associated to a loop in a group acting on a topological space have a long history. Many of them (see [AK, FH, GW, He, N]) are based on a beautiful and quite counter-intuitive idea to look for such skew products in the closure of ones with absolutely trivial dynamical behaviour. ${ }^{3}$ Here is the precise statement in our setting.

Consider the set $C^{\infty}\left(S^{1}, G\right)$ of all smooth loops $S^{1} \rightarrow G$ as a subset of the space $C^{\infty}\left(S^{1} \times Y, Y\right)$, and endow it with the topology induced by the $C^{\infty}$-topology. Consider the subset $\mathcal{R} \subset S^{1} \times C^{\infty}\left(S^{1}, G\right)$ which is defined as the closure of the following subset:

$$
\left\{(\alpha, h) \mid h(t)=g(t+\alpha)^{-1} g(t) \text {, for some } g \in C^{\infty}\left(S^{1}, G\right)\right\} .
$$

Theorem 1.2.B. The pairs $(\alpha, h)$ such that the transformation $(t, y) \rightarrow$ $(t+\alpha, h(t) y)$ is strictly ergodic form a residual subset in $\mathcal{R}$.

Though in the literature there are plenty of similar statements, those of them which I found deal either with other groups $G$, or with usual ergodicity. We outline the proof of 1.2.B in $\S 2$ below, and give full details in $\S 3-\S 5$.

Note that the loops $h$ which appear in the definition of the set $\mathcal{R}$ are limits of contractible loops on $G$ (just let $\alpha$ tend to zero), and therefore are contractible. Thus 1.2.B implies 1.2.A.

\footnotetext{
3 A different approach based on KAM theory was used in [E1, E2], see also 1.7.B below.
} 


\subsection{An obstruction via Hofer's geometry}

Let $Y$ be a closed connected symplectic manifold and let $G$ be the group of all Hamiltonian diffeomorphisms. ${ }^{4}$ In 1990 Hofer $[\mathrm{H}]$ discovered a remarkable bi-invariant geometry on $G$. The development of this geometry has lead to a new intuition in dynamical systems (see discussion in [P2, P3]), and our approach to Question 1.1.A may be considered as a step in this direction. We refer the reader to [P3] for a survey of Hofer's geometry.

Let us introduce the notion of the (asymptotic) length spectrum of $G$ which is relevant for our study of loops of Hamiltonian diffeomorphisms (see [P1]). Every smooth loop $h: S^{1} \rightarrow G$ is generated by the unique Hamiltonian function $H: S^{1} \times Y \rightarrow \mathbf{R}$ which is normalized as follows: the integral of $H(t,$.$) over Y$ vanishes for all $t$. Define the length of the loop $h$ by

$$
\text { length }(h)=\int_{0}^{1} \max _{y \in Y}|H(t, y)| d t \text {. }
$$

Note that $G$ can be considered as an infinite-dimensional Lie group whose Lie algebra coincides with the space $C_{0}^{\infty}(Y)$ of smooth functions with zero mean. The $L_{\infty}$-norm on the Lie algebra is invariant under the adjoint action of $G$, so it defines a bi-invariant Finsler metric on $G$. With this language the definition of the length above is just the usual definition of Finsler length.

Take now an element $\gamma$ of the fundamental group $\pi_{1}(G)$. Set

$$
\|\gamma\|=\inf \text { length }(h) \text {, }
$$

where the infimum is taken over all loops $h: S^{1} \rightarrow G$ representing $\gamma$. Finally, define asymptotic Hofer's norm of $\gamma$ as

$$
\|\gamma\|_{\infty}=\lim _{k \rightarrow \infty} \frac{1}{k}\left\|\gamma^{k}\right\| .
$$

(The limit exists since the sequence $\left\{\| \gamma^{k}||\right\}$ is subadditive).

Using methods of "hard" symplectic topology, one can show that in some interesting situations this quantity is non-trivial (see [P1]).

Theorem 1.3.A. Let $\gamma \in \pi_{1}(G)$ be a class represented by a smooth strictly ergodic loop. Then asymptotic Hofer's norm $\|\gamma\|_{\infty}$ vanishes.

Proof of Theorem 1.1.B. Let $Y$ be the monotone blow up of $\mathbf{C P}^{2}$ at one point as in 1.1.B. It follows from 1.2.A that there exists a contractible strictly ergodic loop. On the other hand, it was shown in [P1] that in this case asymptotic Hofer's norm of every non-trivial element of $\pi_{1}(G)$ is strictly positive. Thus there are no non-contractible strictly ergodic loops in view of Theorem 1.3.A. This completes the proof.

The proof of 1.3.A is very simple and we present it immediately in 1.4.

\footnotetext{
${ }^{4}$ In this section we do not impose any additional assumption on $Y$.
} 


\subsection{Asymptotic shortening of strictly ergodic Hamiltonian loops}

Recall that if $h_{1}(t)$ and $h_{2}(t)$ are paths of Hamiltonian diffeomorphisms generated by normalized Hamiltonians $H_{1}$ and $H_{2}$ respectively then the composition $h_{2}(t) \circ h_{1}(t)$ is generated by the normalized Hamiltonian $H_{2}(t, y)+H_{1}\left(t, h_{2}(t)^{-1} y\right)$. Let $h: S^{1} \rightarrow G$ be a smooth loop of Hamiltonian diffeomorphisms which defines a strictly ergodic skew product $T(t, y)=(t+\alpha, h(t) y)$. Let $\gamma$ be the corresponding element in $\pi_{1}(G)$. Denote by $H(t, x)$ the normalized Hamiltonian function generating the loop $h(t)^{-1}$. Set $h_{k}(t)=h(t+k \alpha)^{-1}$ and set

$$
f_{N}(t)=h_{0}(t) \circ \ldots \circ h_{N-1}(t) .
$$

In view of the discussion above the loop $f_{N}$ is generated by the normalized Hamiltonian function

$$
\begin{aligned}
& F_{N}(t, y)=H(t, y)+H\left(t+\alpha, h_{0}(t)^{-1} y\right)+\ldots \\
& +H\left(t+(N-1) \alpha, h_{N-2}(t)^{-1} \circ \ldots \circ h_{0}(t)^{-1} y\right) .
\end{aligned}
$$

This expression can be rewritten as follows:

$$
F_{N}(t, y)=\Sigma_{k=0}^{N-1} H \circ T^{k}(t, y) .
$$

Since $T$ is strictly ergodic and the function $F_{N}$ has zero mean we conclude that

$$
\frac{1}{N} \int_{0}^{1} \max _{y \in Y}\left|F_{N}(t, y)\right| d t \rightarrow 0,
$$

when $N \rightarrow \infty$. But the expression on the left hand side is exactly $\frac{1}{N}$ length $\left(f_{N}(t)\right)$. Note now that the loop $f_{N}(t)$ represents the element $\gamma^{-N}$. Since $\left\|\gamma^{N}\right\|=\left\|\gamma^{-N}\right\|$ we get that $\frac{1}{N}\left\|\gamma^{N}\right\|$ tends to zero when $N \rightarrow \infty$. This proves that asymptotic Hofer's norm of $\gamma$ vanishes.

\subsection{A generalization to sequential systems}

We present here a generalization of Theorem 1.3.A which deals with ergodic properties of so called sequential dynamical systems. Let $X$ be a compact topological measure space, and let $\left\{T_{i}\right\}=\left(T_{1}, T_{2}, \ldots, T_{i}, \ldots\right)$ be a sequence of measure-preserving homeomorphisms. Such a sequence defines an evolution with discrete time on $X$. Namely a position of a point $x \in X$ at the time moment $n \in \mathbf{N}$ is $T^{(n)}(x)$, where here and below we write $T^{(n)}$ for the composition $T_{n} \circ \ldots \circ T_{1}$. Ergodic properties of such systems were studied in the literature (see for instance [BB], as well as an extensive discussion on random ergodic theorems in $[\mathrm{Kr}])$. However, we have not found any reference to the next definition which sounds to us pretty natural. A sequence 
$\left\{T_{i}\right\}$ is called strictly ergodic if for every continuous function $H$ on $X$ with zero mean the time averages

$$
\frac{1}{N} \Sigma_{i=0}^{N-1} H \circ T^{(i)}
$$

converge uniformly to zero. Our convention is that $T^{(0)}$ is the identity map.

Let as before $G$ be the group of Hamiltonian diffeomorphisms of a closed symplectic manifold $(Y, \Omega)$, and $X=S^{1} \times Y$. Let $\left\{\alpha_{i}\right\}, i \geq 1$ be an arbitrary sequence of numbers, and let $\left\{g_{i}\right\}$ be an arbitrary sequence of Hamiltonian diffeomorphisms. Take a smooth loop $h: S^{1} \rightarrow G$ and consider a sequence $\left\{T_{i}\right\}$ of skew products of the form $T_{i}(t, y)=\left(t+\alpha_{i}, g_{i} h(t) y\right)$. Denote by $\gamma \in \pi_{1}(G)$ the element represented by $h$. In this setting one can generalize Theorem 1.3.A as follows.

If the sequence $T_{i}$ is strictly ergodic then asymptotic Hofer's norm of $\gamma$ vanishes.

This can be proved by the following modification of the shortening procedure described in 1.4 above. Denote by $H(t, y)$ the normalized Hamiltonian function of $h(t)^{-1}$.

Let $\phi_{0}=$ id, $\phi_{1}, \phi_{2}, \ldots$ be a sequence of transformations from $G$ such that $\phi_{i}^{-1} \phi_{i-1}=g_{i}$ for all $i \geq 1$. Set

$$
h_{k}(t)=\phi_{k} h\left(t+\alpha_{0}+\ldots+\alpha_{k}\right)^{-1} \phi_{k}^{-1},
$$

where $\alpha_{0}=0$. Consider a new loop

$$
f_{N}(t)=h_{0}(t) \circ \ldots \circ h_{N-1}(t) .
$$

It is easy to see that $f_{N}$ is generated by the normalized Hamiltonian

$$
F_{N}=\Sigma_{i=0}^{N-1} H \circ T^{(i)},
$$

and this loop represents the class $\gamma^{-N}$. Now exactly the same argument as in 1.4 completes the proof.

\subsection{An application to Hofer's geometry}

I do not know the precise value of $\|\gamma\|_{\infty}$ in any example where this quantity is strictly positive (for instance, for the blow up of $\mathbf{C P}^{2}$ in 1.1 above). The difficulty is as follows. In all known examples where Hofer's norm $\|\gamma\|$ can be computed precisely there exists a closed loop $h(t)$ which minimizes the length in its homotopy class (that is a minimal closed geodesic). It turns out however that every non-constant minimal closed geodesic loses minimality after a suitable number of iterations. In other words the loop $h(N t)$ can be shortened provided $N$ is large enough. The proof of this statement is based 
on a shortening procedure described in the previous section and goes as follows.

Let $H(t, y)$ be the normalized Hamiltonian of $h(t)^{-1}$. Assume without loss of generality that $h(0)=\mathrm{id}$, and that $H(0, y)$ does not vanish identically. Denote by $\Gamma$ the set of all points of $Y$ where the function $|H(0, y)|$ attains the maximal value. Since $Y-\Gamma$ is a non-empty open subset, and the group $G$ acts transitively on $Y$, one can choose a sequence $\phi_{0}=\mathrm{id}, \phi_{1}, \ldots, \phi_{N-1} \in G$ such that

$$
\Gamma \cap \phi_{1}(\Gamma) \cap \ldots \cap \phi_{N-1}(\Gamma)=\emptyset .
$$

Consider the loop $f_{N}(t)=h(t)^{-1} \circ \phi_{1} h(t)^{-1} \phi_{1}^{-1} \circ \ldots \circ \phi_{N-1} h(t)^{-1} \phi_{N-1}^{-1}$. We claim that it is shorter than the loop $h(N t)$. Indeed, note that its Hamiltonian $F_{N}$ at time $t=0$ can be written as follows:

$$
F_{N}(0, y)=\Sigma_{i=0}^{N-1} H\left(0, \phi_{i}^{-1} y\right) .
$$

Set $a(t)=\max _{y \in Y}\left|F_{N}(t, y)\right|$ and $b(t)=N \max _{y \in Y}|H(t, y)|$. Our choice of the sequence $\left\{\phi_{i}\right\}$ implies that $a(0)<b(0)$. Since $a(t) \leq b(t)$ for all $t$, we get that $\int_{0}^{1} a(t) d t<\int_{0}^{1} b(t) d t$, and this proves the claim. We conclude that if a non-zero class $\gamma \in \pi_{1}(G)$ is represented by a minimal geodesic then $\|\gamma\|_{\infty}$ is strictly less than $\|\gamma\|$.

Let us complete this section with few remarks on curve shortening in Hofer's geometry. The first shortening procedure is due to Sikorav [Si]. Further progress was made by Ustilovsky [U], Lalonde - McDuff [LM], and in a joint paper with Bialy [BP]. Our procedures in 1.4 and 1.5 are closely related to these developments. In particular, in [BP] we asked a question about the role of Birkhoff's ergodic sums in Hofer's geometry. The results above can be considered as a sort of answer.

\subsection{Remarks and open problems}

1.7.A. Further obstructions? Do there exist further restrictions on the homotopy classes of smooth strictly ergodic loops in the group of Hamiltonian diffeomorphisms? I do not know the answer even in the simplest case when $Y$ is the 2-sphere endowed with an area form. In this case the group of Hamiltonian diffeomorphisms has the homotopy type of $S O(3)$, and thus its fundamental group equals $\mathbf{Z}_{2}$. It would be interesting to understand whether in this situation there exists a smooth strictly ergodic loop in the non-trivial homotopy class. Note that the obstruction provided by Theorem 1.3.A cannot be applied since the homotopy class in question has finite order.

1.7.B. Continuous vs. smooth. Question 1.1.A still makes sense if one considers continuous loops of Hamiltonian diffeomorphisms instead of smooth ones. In this situation the existence result 1.2.A above can be refined as 
follows. One can show existence of contractible continuous strictly ergodic loops with every given irrational rotation number $\alpha$. Note that in the smooth case the methods used below lead to those $\alpha$ 's only which admit a very fast approximation by rationals. ${ }^{5}$ On the other hand, our proof of the obstruction 1.3. A above does not go through when a strictly ergodic loop is continuous, since it crucially uses existence of the Hamiltonian function.

1.7.C. The volume-preserving case. Let $Y$ be a closed manifold endowed with a volume form and let $G$ be the identity component of the group of all volume-preserving diffeomorphisms. Exactly as in the Hamiltonian case, one can address the question about homotopy classes represented by strictly ergodic loops. The formulation and the proof of the existence result 1.2.A remain valid without any changes in the volume-preserving category. However strictly ergodic loops may well be non-contractible. We present here an example of a non-contractible strictly ergodic loop in the group of area preserving diffeomorphisms of the 2-torus. Note that in dimension 2 every area preserving diffeomorphism is symplectic but not necessarily Hamiltonian. This leads to a suggestion that the phenomenon described in 1.3.A is a purely Hamiltonian one.

Take irrational numbers $\alpha$ and $\beta$ such that $1, \alpha$ and $\beta$ are independent over $\mathbf{Q}$. Consider the loop of transformations of the 2-torus $h(t): \mathbf{T}^{2} \rightarrow \mathbf{T}^{2}$ which take $\left(y_{1}, y_{2}\right) \in \mathbf{T}^{2}$ to $\left(y_{1}+t, y_{2}+\beta\right)$. Clearly these transformations preserve the area form $d y_{1} \wedge d y_{2}$ on $\mathbf{T}^{2}$, and the loop is not contractible. The standard harmonic analysis argument shows that the corresponding skew product $T_{h, \alpha}: \mathbf{T}^{3} \rightarrow \mathbf{T}^{3}$ is ergodic. It follows from a theorem due to Furstenberg [F2, p. 66] that in this case $T_{h, \alpha}$ is strictly ergodic.

Moreover, in the case of volume-preserving diffeomorphisms of the circle $Y=S^{1}$ Furstenberg [F1] observed an opposite phenomenon: "noncontractibility implies strict ergodicity". In this situation every non-contractible loop $h: S^{1} \rightarrow S^{1} \subset G$ is strictly ergodic.

\section{Constructing strictly ergodic skew products}

Let $\mu$ be the canonical measure on $Y$. We write $\mathcal{H}$ for the space of all continuous functions on $Y$ with zero mean with respect to $\mu$. This space is endowed with a norm $\|H\|=\max _{y \in Y}|H(y)|$. Recall that our task is to prove Theorem 1.2.B on the existence of smooth strictly ergodic loops.

\footnotetext{
5 This feature of the classical approach (see $\$ 2$ below) is well known to experts. It sounds likely however that using methods developed by Eliasson [E1, E2] one can construct strictly ergodic Hamiltonian skew products on $S^{1} \times S^{2}$ whose rotation numbers satisfy a diophantine condition.
} 


\subsection{The classical approach}

The proof of 1.2.B is based on the following chain of statements.

Property 2.1.A. For every continuous function $F: S^{1} \times Y \rightarrow \mathbf{R}$ with zero mean with respect to $d t d \mu$, for every $\varepsilon>0$ and for every rational number $r$ there exists a loop $g \in C^{\infty}\left(S^{1}, G\right)$ such that the following two conditions hold:

(i) $\left|\int_{0}^{1} F\left(t, g(t)^{-1} y\right) d t\right|<\varepsilon$ for all $y \in Y$;

(ii) $g(t+r)=g(t)$ for all $t$.

Averaging property 2.1.B. For every $H \in \mathcal{H}$ and $\varepsilon>0$ there exist transformations $g_{1}, \ldots, g_{N} \in G$ such that

$$
\frac{1}{N}\left|H\left(g_{1}^{-1} y\right)+\ldots+H\left(g_{N}^{-1} y\right)\right|<\varepsilon,
$$

for all $y \in Y$.

Note that 2.1.B is a natural discrete version of 2.1.A(i). However in contrast with 2.1.A we consider here functions $H$ of the variable $y$ only, and do not care about the commutativity condition 2.1.A(ii).

Property 2.1.A implies the statement of Theorem 1.2.B (see [FH]). Averaging property 2.1.B implies property 2.1.A (see $[\mathrm{N}]$ where an analogous implication is proved in the context of ergodicity; in our situation the argument goes through without any essential modifications). For the reader's convenience, we present details in the Appendix in $\$ 5$.

At this point we face a difficulty. The analogue of 2.1.B for the $L_{1}$-norm on $\mathcal{H}$, which is used in $[\mathrm{N}]$, was proved earlier by $\mathrm{M}$. Herman [He] with a very elegant use of the Hahn-Banach theorem. I was unable to adjust Herman's short argument to the $L_{\infty}$-case, and thus was forced to take a different route. The key idea is to derive the averaging property from a certain covering property which we are going to describe now.

Let us introduce the following useful object. Denote by $\mathcal{S}$ the set of linear operators $\mathcal{H} \rightarrow \mathcal{H}$ which consists of all averaging operators of the form

$$
S^{g_{1}, \ldots, g_{N}}(H)=\frac{1}{N}\left(H \circ g_{1}^{-1}+\ldots+H \circ g_{N}^{-1}\right),
$$

where $N \in \mathbf{N}$ and $g_{1}, \ldots, g_{N} \in G$. Note that $\mathcal{S}$ is closed under composition of operators. With this notation 2.1.B states that for all $H \in \mathcal{H}$ there exists $S \in \mathcal{S}$ such that $\|S(H)\|$ is arbitrarily small. An important (and obvious) feature of transformations from $\mathcal{S}$ is that they do not increase the norm of functions: $\|S(H)\| \leq\|H\|$. 


\subsection{A covering property}

Covering property 2.2.A. There exist constants $c_{1} \geq 0, c_{2} \geq 1$ such that for every non-empty open subset $A \subset Y$ one can find transformations $g_{1}, \ldots, g_{N} \in G$ so that the sets $g_{1}(A), \ldots, g_{N}(A)$ form a covering of $Y$ which satisfies the following inequality:

$$
\frac{1}{N} \Sigma_{i=1}^{N} \chi^{g_{i}(A)}(y) \geq\left(c_{1}+c_{2} \frac{\mu(Y)}{\mu(A)}\right)^{-1},
$$

for all $y \in Y$.

Here and below $\chi^{B}$ stands for the characteristic function of a subset $B$.

Theorem 2.2.B. The covering property implies the averaging property.

Note that averaging property 2.1.B applied to the normalized characteristic function of an open subset $A$ implies up to $\varepsilon$ covering property 2.2.A with the optimal constants $c_{2}=1$ and $c_{1}=0$. Hence a surprising feature of Theorem 2.2.B is that starting from an arbitrary choice of the constants we get the optimal constants. Let us mention also that our covering property is motivated by the Glasner-Weiss covering property [GW].

The rest of the paper is organized as follows. Theorem 2.2.B admits a rather short proof which we present in Sect. 3. Thus it remains to verify that the group of Hamiltonian diffeomorphisms enjoys covering property 2.2.A. Here is the idea of our proof. "Represent" the symplectic manifold $Y$ as a cubical polyhedron consisting of small symplectically standard pairwise equal closed cubes. There exists a universal constant, say $k$ which depends only on $Y$ (but not on the size of the cubes!) such that $Y$ can be decomposed as the union of subpolyhedra $Y_{1}, \ldots, Y_{k}$ where each $Y_{i}$ consists of cubes with pairwise disjoint closure. Assume without loss of generality that the set $A$ given in 2.2.A is a subpolyhedron of $Y$, and $\mu\left(A \cap Y_{1}\right) \geq \mu(A) / k$. Set $A_{1}=$ $A \cap Y_{1}$, and assume that this set consists of $m$ cubes. Assume for simplicity that each $Y_{i}$ consists of $M$ cubes with $M>m$. Clearly, every subpolyhedron of $Y_{i}$ which consists of $m$ cubes is Hamiltonian diffeomorphic to $A_{1}$ (cf. [HZ, p. 171]). Denote by $r$ the number of all such subpolyhedra in $Y_{i}$, thus their total number is $N=k r$. Note that every point of $Y_{i}$ belongs to at least $\mathrm{rm} / M$ subpolyhedra from our collection. Thus we can choose $N$ elements of $G$ such that for every point of $Y$ the left hand side of the inequality 2.2.A is at least

$$
r m / M k r=m / M k \geq \mu(A) / k \mu(Y) .
$$

Since $k$ is a universal constant, we get 2.2.A. The details of this elementary argument are quite cumbersome. They are worked out in Sect. 4 with the use of Katok's results [K]. This will complete the proof of Theorem 1.2.B. 


\section{Covering implies averaging}

In this section we prove Theorem 2.2.B.

\subsection{A recursive procedure}

Suppose that covering property 2.2.A holds. We have to show that for all $H \in \mathcal{H}$ there exists $S \in \mathcal{S}$ such that $\|S(H)\|$ is arbitrarily small.

We claim that it suffices to show that there exists $S_{+} \in \mathcal{S}$ such that $\max S_{+}(H)$ is arbitrarily small. Indeed, note that the same result applied to the function $-S_{+}(H)$ would show that there exists $S_{-} \in \mathcal{S}$ such that $\min S_{-}\left(S_{+}(H)\right)$ is arbitrarily small. But obviously operators from $\mathcal{S}$ do not increase maximal values of functions, thus $\max S_{-}\left(S_{+}(H)\right) \leq \max S_{+}(H)$. So taking $S=S_{-} \circ S_{+}$we get that $\|S(H)\|$ is arbitrarily small. The claim follows.

Assume without loss of generality that $\|H\| \leq 1$ and $\mu(Y)=1$. We construct the operator $S_{+}$with the help of the following recursive procedure. We start with the function $H^{(0)}=H$, and define $H^{(i+1)}$ as the image of $H^{(i)}$ under some specially chosen operator $S_{i} \in \mathcal{S}$. Namely consider a subset

$$
A_{i}=\left\{H^{(i)}<\frac{1}{2} \max H^{(i)}\right\} \subset Y .
$$

Take $g_{1}, \ldots, g_{N}$ from the definition of the covering property 2.2.A applied to the set $A_{i}$, and set $S_{i}=S^{g_{1}, \ldots, g_{N}}$.

Lemma 3.1.A. The following inequality holds:

$$
\max H^{(i+1)} \leq \max H^{(i)}\left(1-\frac{\max H^{(i)}}{c}\right),
$$

where $c=2\left(3 c_{2}+c_{1}\right)$.

The theorem easily follows from the lemma. We claim that the operator $S_{+}=S_{i} \circ \ldots \circ S_{1}$ does the job provided $i$ is large enough. Indeed, set $m_{i}=\max H^{(i)}$. Notice that $m_{0} \leq 1$ due to our assumption, and the sequence $\left\{m_{i}\right\}$ is non-increasing since operators from $\mathcal{S}$ do not increase maximal values of functions. Clearly the sequence $\left\{m_{i}\right\}$ converges to a non-negative number $m$. For the proof of the claim (and hence of the theorem) it suffices to show that $m=0$. Assume on the contrary that $m>0$. Lemma 3.1.A implies that $m \leq m\left(1-\frac{m}{c}\right)$, which is obviously impossible. This contradiction proves the theorem. 


\subsection{Proof of 3.1.A}

For simplicity of notations we write $H$ for $H^{(i)}, H^{\prime}$ for $H^{(i+1)}$, and $A$ for $A_{i}$. Set $m=\max H$ and $m^{\prime}=\max H^{\prime}$. The proof is divided into 2 parts.

1) Our first task is to find a lower bound for $\mu(A)$. Since $H$ has zero mean we have

$$
\int_{A} H d \mu+\int_{Y-A} H d \mu=0 .
$$

The first summand is not less than $-\mu(A)$ since $\|H\| \leq 1$ due to our assumption. The second summand is not less than $\frac{1}{2} m(1-\mu(A))$ in view of the definition of $A$ and our convention that $\mu(Y)=1$. Thus $0 \geq-\mu(A)+$ $(1-\mu(A)) m / 2$ and hence

$$
\mu(A) \geq m /(m+2) .
$$

2) Return now to the definition of $H^{\prime}$. Take a point $y \in Y$ and denote by $N^{\prime}$ the cardinality of the set $\left\{j \mid y \in g_{j}(A)\right\}$. Clearly,

$$
H^{\prime}(y) \leq \frac{1}{N}\left(\left(N-N^{\prime}\right) m+N^{\prime} m / 2\right)=m\left(1-\frac{N^{\prime}}{2 N}\right) .
$$

Since this holds for all $y$, the maximum $m^{\prime}$ of $H^{\prime}$ satisfies the same inequality. On the other hand the covering property 2.2.A implies that

$$
N^{\prime} / N \geq\left(c_{1}+c_{2} / \mu(A)\right)^{-1} .
$$

Substituting the inequality for $\mu(A)$ obtained in part 1 , we get that

$$
N^{\prime} / N \geq m /\left(2 c_{2}+\left(c_{1}+c_{2}\right) m\right) \geq m / c_{3},
$$

where $c_{3}=3 c_{2}+c_{1}$. In the last inequality we used that $m \leq 1$. Finally, substituting this estimate for $N / N^{\prime}$ into the estimate for $m^{\prime}$ obtained above, we get that $m^{\prime} \leq m(1-m / c)$. This completes the proof.

\section{Proving the covering property}

\subsection{Statement of the result}

In this section we prove the following result. As before, let $G$ be the group of Hamiltonian diffeomorphisms of a closed symplectic manifold $(Y, \Omega)$.

Theorem 4.1.A. The group $G$ enjoys covering property 2.2.A.

As it was explained in $\S 2$, this result completes the proof of Theorem 1.2.B. 


\subsection{A local version}

First of all we prove an analogue of 4.1.A for domains of the even dimensional linear space $\mathbf{R}^{n}$. We assume that $\mathbf{R}^{n}$ is endowed with the standard symplectic form. Let $X \subset \mathbf{R}^{n}$ be a closed bounded connected domain with piece-wise smooth boundary. Let $U$ be an open domain with compact closure which contains $X$, and write $G_{U}$ for the group of all Hamiltonian diffeomorphisms generated by Hamiltonian functions supported in $U$. We denote by $\mu$ the canonical measure on $\mathbf{R}^{n}$. Also, given a family $\theta=\left\{A_{1}, \ldots, A_{N}\right\}$ of subsets of $U$, we write $v_{\theta}$ for the "counting function" $\sum_{i=1}^{N} \chi^{A_{i}}$.

Proposition 4.2.A. There exist universal constants $C_{1}=C_{1}(n) \geq 0$, $C_{2} \geq 1$ such that for every non-empty open subset $A$ of the interior of $X$ there exist transformations $g_{1}, \ldots, g_{M} \in G_{U}$ such that $\theta=\left\{g_{1}(A), \ldots, g_{M}(A)\right\}$ is a covering of $X$, and

$$
\frac{1}{M} v_{\theta}(x) \geq\left(C_{1}+C_{2} \frac{\mu(X)}{\mu(A)}\right)^{-1},
$$

for all $x \in X$.

The proof is based on two auxiliary statements.

Lemma 4.2.B. For every $a \in(0 ; \mu(X)]$ there exists a family $\sigma=\left\{A_{1}, \ldots\right.$, $\left.A_{N}\right\}$ of open subsets of $U$ with compact closure in $U$ such that $\mu\left(A_{j}\right)=a$ and $v_{\sigma}(x) / N \geq a / 2 \mu(X)$, for all $x \in X$.

Lemma 4.2.C. There exists a constant $C \in \mathbf{N}$ which depends only on the dimension of $X$ with the following property. Let $A, B \subset U$ be two open subsets with compact closure in $U$ such that $\mu(A)>2 C \mu(B)$. Then there exist $C$ transformations $g_{1}, \ldots, g_{C} \in G_{U}$ such that $B \subset \cup_{i=1}^{C} g_{i}(A)$.

Let us derive Proposition 4.2.A from these lemmas.

Proof of 4.2.A. Take any open subset $A$ as in 4.2.A and apply Lemma 4.2.B with $a=\mu(A)$. We get a family $\sigma=\left\{A_{1}, \ldots, A_{N}\right\}$ of subsets. Fix $\varepsilon>0$. We claim that there exist $f_{1}, \ldots, f_{N} \in G_{U}$ such that $\mu\left(f_{i}(A) \Delta A_{i}\right)<\varepsilon / N$ for all $i=1, \ldots, N$. Here $\Delta$ stands for the symmetric difference of subsets. The claim follows immediately from Katok's Basic Lemma $[\mathrm{K}]^{6}$. Consider a family of subsets $\tau=\left\{f_{1}(A), \ldots, f_{N}(A)\right\}$, and set $B=\cup_{i=1}^{N}\left(f_{i}(A) \Delta A_{i}\right)$. Obviously, $\mu(B)<\varepsilon$. Assume now that $\varepsilon$ is so small that Lemma 4.2.C can

\footnotetext{
6 For the reader's convenience we present the formulation of the lemma. Let $\left\{D_{i}\right\}, \quad\left\{D_{i}^{\prime}\right\}, i=1, \ldots, N$ be two collections of compact subsets of a connected symplectic manifold. Assume that the sets in each collection are pair-wise disjoint, and the volume of $D_{i}$ equals the volume of $D_{i}^{\prime}$ for all $i$. Then for every $\varepsilon>0$ there exists a Hamiltonian diffeomorphism $\phi$ such that Volume $\left(\phi\left(D_{i}\right) \Delta D_{i}^{\prime}\right) \leq \varepsilon$ for all $i$.
} 
be applied to $A$ and a small open neighbourhood of $B$. Using this lemma, we get $C$ transformations $g_{1}, \ldots, g_{C} \in G_{U}$ such that $B \subset \cup g_{i}(A)$.

Set $N^{\prime}=\min _{x \in X} v_{\sigma}(x)$. Define a new family $\theta$ which consists of all subsets of the form $g_{i}(A), i=1, \ldots, C$ taken $N^{\prime}$ times, and in addition of all subsets from $\tau$. In other words,

$$
\theta=\left\{f_{1}(A), \ldots, f_{N}(A) ; g_{1}(A), \ldots, g_{C}(A), \ldots, g_{1}(A), \ldots, g_{C}(A)\right\} .
$$

The number $M$ of elements in $\theta$ equals to $N+C N^{\prime}$. On the other hand, we claim that $v_{\theta}(x) \geq N^{\prime}$ for all $x \in X$. Indeed, for $x \in B$ this follows from the definition of transformations $g_{i}$. For $x \in X-B$ we note that $v_{\tau}(x)=v_{\sigma}(x)$ and the claim follows from the definition of $N^{\prime}$.

Recall now from Lemma 4.2.B that $N^{\prime} / N \geq \mu(A) / 2 \mu(X)$. Thus

$$
\frac{1}{M} v_{\theta} \geq N^{\prime} /\left(N+C N^{\prime}\right) \geq\left(C+2 \frac{\mu(X)}{\mu(A)}\right)^{-1} .
$$

Therefore we proved 4.2.A with $C_{1}=C$ and $C_{2}=2$.

It remains to prove the lemmas. Both are of combinatorial nature, and we need some suitable notions. By a cubical partition of size $u$ we mean a decomposition of $\mathbf{R}^{n}$ into equal closed cubes of volume $u$. The cubes may intersect along the boundaries only, and their centers form a lattice. A cubical polyhedron is the union of some cubes of a cubical partition.

Proof of 4.2.B. Take a sufficiently large positive integer $k$ and consider a cubical partition of size $a / k$. We can assume that there exists a cubical polyhedron $X^{\prime}, \quad X \subset X^{\prime} \subset U$ such that $\mu\left(X^{\prime}\right)<1.5 \mu(X)$. Take all possible sub-polyhedra of $X^{\prime}$ consisting of exactly $k-1$ cubes. Let $A_{1}, \ldots, A_{N}$ be their open neighbourhoods of volume $a$ which have compact closure in $U$. Denote this family by $\sigma$. Clearly for all $x \in X$

$$
v_{\sigma}(x) / N \geq(k-1) a / k \mu\left(X^{\prime}\right) \geq a / 2 \mu(X) .
$$

This proves the lemma.

For the proof of the second lemma we need the following facts from elementary geometry of $\mathbf{R}^{n}$. Given a closed cube $Q \subset \mathbf{R}^{n}$ and a positive number $c$ denote by $c Q$ the cube with the same center which is homothetic to $Q$ with the coefficient $c$. Let $Q_{1}, Q_{2}$ be two cubes which are obtained from each other by a translation. Note first that if $Q_{1} \cap Q_{2} \neq \emptyset$ then $Q_{1} \subset$ Interior $\left(4 Q_{2}\right)$. Define a nice subpartition of a cubical partition as a family of pairwise disjoint cubes from the partition which satisfies the following property. Given any two cubes $Q_{1}$ and $Q_{2}$ from the family, their homothetic images $16 Q_{1}$ and $16 Q_{2}$ are also disjoint. Note finally that there exists a constant $C \in \mathbf{N}$ which depends only on $n$ such that every cubical partition of $\mathbf{R}^{n}$ can be decomposed into $C$ nice subpartitions. 
Proof of 4.2.C.

1) Let $U^{\prime} \subset U$ be an open connected domain with compact closure in $U$ which contains both $A$ and $B$. Fix a cubical partition $P$ of size $u$. We assume that $u$ is so small that for each cube $Q$ of the partition which intersects $U^{\prime}$ holds $16 Q \subset U$. Let $P=P_{1} \cup \ldots \cup P_{C}$ be its decomposition into nice subpartitions. Denote by $A_{i}$ the union of all cubes from $P_{i}$ which are contained in $A$, and by $B_{i}$ the union of all cubes from $P_{i}$ which intersect $B$. Clearly taking $u$ small enough we can achieve that $\mu\left(B_{i}\right) \leq 1.1 \mu(B)$ for all $i$, and $\mu\left(A_{i}\right) \geq \mu(A) / 1.1 C$ for some $i$. Assume without loss of generality that the last inequality holds for $i=1$. Since $\mu(A)>2 C \mu(B)$ we conclude that the number of cubes in $A_{1}$ is greater than the number of cubes in each of $B_{i}$. Fix some $i \in\{1, \ldots, C\}$. Clearly, in order to prove the lemma it suffices to show that if $u$ is small enough there exists a transformation $g_{i} \in G$ such that $B_{i} \subset g_{i}\left(A_{1}\right)$.

2) Denote by $q=\left\{Q_{1}, \ldots, Q_{k}\right\}$ the set of all cubes in $A_{1}$, by $q^{\prime}=$ $\left\{Q_{1}^{\prime}, \ldots, Q_{m}^{\prime}\right\}$ the set of all cubes in $B_{i}$, and by $p$ the union $q \cup q^{\prime}$. We write $4 q$ for $\left\{4 Q_{1}, \ldots, 4 Q_{k}\right\}$, and define analogously $4 q^{\prime}$ and $4 p$. Let $Z$ be the subset of $U$ obtained by the union of all cubes from $4 p$. We claim that each connected component of $Z$ is either one cube, or the union of two cubes. Assume on the contrary that there exist three cubes from $4 p$ such that two of them intersect the third one. By definition of a nice subpartion, either these two belong to $4 q$ and the third belongs to $4 q^{\prime}$, or vice versa the two belong to $4 q^{\prime}$ and the third to $4 q$. Without loss of generality we assume that $4 Q_{1}$ and $4 Q_{2}$ intersect $4 Q_{1}^{\prime}$. But then $16 Q_{1}$ and $16 Q_{2}$ contain $4 Q_{1}^{\prime}$, and that contradicts the definition of a nice subpartition. The claim follows.

3 ) In view of step 2, we may assume without loss of generality that for some $r \leq m$

$$
4 Q_{1} \cap 4 Q_{1}^{\prime} \neq \emptyset, \ldots, 4 Q_{r} \cap 4 Q_{r}^{\prime} \neq \emptyset,
$$

and all other pairs of cubes from $4 p$ are disjoint. Recall also that $m<k$. We claim that for every $j \in\{1, \ldots, m\}$ there exists a transformation $h_{j} \in G_{U}$ such that $h_{j}\left(Q_{j}\right)=Q_{j}^{\prime}$ and $h_{j}$ equals the identity on all $Q_{l}, Q_{l}^{\prime}$ with $l \neq j$. Note that this claim implies the lemma. Indeed set $g_{i}=h_{1} \circ \ldots \circ h_{r}$. Clearly $g_{i}\left(Q_{j}\right)=Q_{j}^{\prime}$, and hence we constructed a transformation as required in step 1.

4) It remains to prove the claim of step 3 . Take $j \in\{1, \ldots, m\}$.

First assume that $j \leq r$. Consider a set $K=16 Q_{j} \cap 16 Q_{j}^{\prime}$. Clearly, $K$ is a convex polyhedron whose interior contains both $Q_{j}$ and $Q_{j}^{\prime}$. There exists a path of transformations from $G_{U}$ supported in $K$ whose time one map takes $Q_{j}$ to $Q_{j}^{\prime}$. Take this time one map as $h_{j}$. Any other cube from $p$ is disjoint from $K$ by definition of a nice subpartition. Thus $h_{j}$ has the required properties. 
Assume now that $j>r$. Consider the set $Z_{j}=Z-\left(4 Q_{j} \cup 4 Q_{j}^{\prime}\right)$. It follows from step 2 that $U^{\prime}-Z_{j}$ is a connected set. Join the centers of cubes $Q_{j}$ and $Q_{j}^{\prime}$ by a smooth path $\gamma \subset U^{\prime}-Z_{j}$. Let $t$ be a parameter along $\gamma$ which runs from 0 to 1 . Denote by $K_{t}$ the cube centered in $\gamma(t)$ which is obtained from $Q_{j}$ by a parallel translation. Since the center of $K_{t}$ is disjoint from all cubes $4 Q_{l}, 4 Q_{l}^{\prime}$ with $l \neq j$ then $K_{t}$ is disjoint from all such $Q_{l}$ and $Q_{l}^{\prime}$. In particular, there exists a small neighbourhood say $V$ of the union of all cubes $K_{t}$ which is disjoint from all cubes $Q_{l}, Q_{l}^{\prime}$ with $l \neq j$. It is easy to see that there exists a path of transformations from $G_{U}$ supported in $V$ whose time one map takes $Q_{j}$ to $Q_{j}^{\prime}$. Take this time one map as $h_{j}$. This completes the proof.

\subsection{Proof of 4.1.A}

Denote by $\mu$ the canonical measure on $Y$. Consider a triangulation $X_{1}, \ldots, X_{r}$ of $Y$ such that every simplex $X_{i}$ is contained in a (Darboux) coordinate chart $U_{i}$. Assume moreover that all $X_{i}$ have equal volume $\mu(Y) / r$. Let $A \subset Y$ be an open subset. A straightforward application of Katok's Basic Lemma ([K], see the footnote in 4.2 above) shows that there exists a transformation $f \in G$ with the following property:

$$
\mu\left(f(A) \cap X_{i}\right)>\mu(A) / 2 r,
$$

for all $i=1, \ldots, r$.

Obviously it suffices to check the covering property for the set $A^{\prime}=$ $f(A)$. Let $A_{i}$ be an open set which lies in the interior of $A^{\prime} \cap X_{i}$ and has volume $\mu(A) / 2 r$. Apply the local statement 4.2.A to the triple $\left(U_{i}, X_{i}, A_{i}\right)$. For every $i$, we get a sequence of transformations $g_{i j}, j=1, \ldots, N_{i}$ such that for all $x \in X_{i}$ holds

where

$$
\frac{1}{N_{i}} \Sigma_{j=1}^{N_{i}} \chi^{g_{i j}\left(A_{i}\right)}(x) \geq\left(C_{1}+C_{2} \frac{\mu\left(X_{i}\right)}{\mu\left(A_{i}\right)}\right)^{-1}=\lambda,
$$

$$
\lambda=\left(C_{1}+2 C_{2} \frac{\mu(Y)}{\mu\left(A^{\prime}\right)}\right)^{-1} .
$$

Repeating the sequences we can achieve that each of them has the same number of terms, in other words that all $N_{i}$ 's are equal to the same number $N$. We claim that the family of transformations

$$
\left\{g_{i j}\right\}, i=1, \ldots, r ; j=1, \ldots, N
$$

does the job for the set $A^{\prime}$ with universal constants $c_{1}=r C_{1}$ and $c_{2}=2 r C_{2}$. Indeed, take $y \in Y$. Without loss of generality assume that $y \in X_{1}$. Thus

$$
\frac{1}{N r} \Sigma_{i, j} \chi^{g_{i j}\left(A^{\prime}\right)}(y) \geq \frac{1}{N r} \Sigma_{j} \chi^{g_{1 j}\left(A_{1}\right)}(y) \geq \frac{\lambda N}{r N}=\left(r C_{1}+2 r C_{2} \frac{\mu(Y)}{\mu\left(A^{\prime}\right)}\right)^{-1} .
$$


This completes the proof.

\section{APPENDIX: more details on the classical approach}

In this appendix we present details of the classical approach to constructing strictly ergodic Hamiltonian skew products (see Sect. 2.1 of the main text).

\subsection{Averaging property 2.1.B implies 2.1.A (cf. [N])}

We work in the notations of Sect. 2.1. The proof is divided into several steps.

1) We claim that for every finite sequence $H_{1}, \ldots, H_{k}$ of functions from $\mathcal{H}$ and for every $\varepsilon>0$ there exists an operator $S \in \mathcal{S}$ such that $\left\|S\left(H_{i}\right)\right\|<\varepsilon$ for all $i$. Indeed, using averaging property 2.1.B define recursively a sequence of operators $S_{i} \in \mathcal{S}$ such that the following inequalities hold:

$$
\left\|S_{1}\left(H_{1}\right)\right\|<\varepsilon,\left\|S_{2}\left(S_{1}\left(H_{2}\right)\right)\right\|<\varepsilon, \ldots, \| S_{k}\left(\ldots\left(S_{1}\left(H_{k}\right) \ldots\right) \|<\varepsilon .\right.
$$

Set $S=S_{k} \circ \ldots \circ S_{1}$. The operator $S$ is as required in view of the fact that operators from $\mathcal{S}$ do not increase the norm of functions.

As an immediate consequence of the claim we get that for every sequence $H_{1}, \ldots, H_{k}$ and every $\varepsilon>0$ there exists a smooth loop $h: S^{1} \rightarrow G$ such that

$$
\left|\int_{0}^{1} H_{i}\left(h(t)^{-1} y\right) d t\right|<\varepsilon
$$

for all $y \in Y$.

2) Note that in order to verify condition 2.1.A(i) we can assume that for each fixed $t$ the function $F(t,$.$) belongs to the space \mathcal{H}$. Indeed, given any $F$ one can modify it as follows:

$$
F^{\prime}(t, y)=F(t, y)-\int_{Y} F(t, z) d \mu(z) .
$$

Clearly our assumption holds for $F^{\prime}$. Moreover $F$ and $F^{\prime}$ satisfy or do not satisfy the conditions in question simultaneously.

3 ) Let $F$ be a function which satisfies the assumption of step 2. We claim that for every $\varepsilon>0$ there exists a smooth loop $h$ such that for all $s \in S^{1}$ the following inequality holds:

$$
\left|\int_{0}^{1} F\left(s, h(t)^{-1} y\right) d t\right|<\varepsilon / 3 .
$$


Indeed, choose a large natural number $N$ such that $\left|F\left(t^{\prime}, y\right)-F\left(t^{\prime \prime}, y\right)\right|<\varepsilon / 9$ for all $y$ provided $\left|t^{\prime}-t^{\prime \prime}\right|<1 / N$. Set $p_{i}=i / N$ where $i=0, \ldots, N-1$. Using the last statement of step 1 choose a smooth loop $h(t)$ such that

$$
\left|\int_{0}^{1} F\left(p_{i}, h(t)^{-1} y\right) d t\right|<\varepsilon / 9 .
$$

A straightforward estimate shows that $h$ is as required.

4) Take now an arbitrary integer $M>N$. A more specific choice of $M$ will be made in the next step. Meanwhile we denote $g(t)=h(M t)$ and claim that

$$
I=\left|\int_{0}^{1} F\left(t, g(t)^{-1} y\right) d t\right|<\varepsilon
$$

Here is the proof. Set $q_{i}=i / M$ where $i=0, \ldots, M$. An obvious argument shows that

$$
I \leq \varepsilon / 3+\Sigma_{i=0}^{M-1}\left|J_{i}\right|
$$

where

$$
J_{i}=\int_{q_{i}}^{q_{i+1}} F\left(q_{i}, h(M t)^{-1} y\right) d t .
$$

Introducing a new variable $s=M t-i$ we get that

$$
J_{i}=\frac{1}{M} \int_{0}^{1} F\left(q_{i}, h(s)^{-1} y\right) d s,
$$

and thus our choice of $h$ implies that $\left|J_{i}\right|<\varepsilon /(3 M)$ for all $i$. The claim follows immediately.

5) Let us sum up the results of the previous steps. We constructed a loop $g(t)=h(M t)$ which satisfies 2.1.A(i) above. Moreover the choice of the sufficiently large integer $M$ is in our hands. We are going to use this in order to guarantee condition 2.1.A(ii). Let $r$ be a rational number. Taking $M$ as a large multiple of the denominator of $r$ we get that $g(t+r)=g(t)$.

This completes the proof.

\subsection{Property 2.1.A implies Theorem 1.2.B}

The proof of this statement occupies the rest of the appendix. We follow very closely the exposition in $[\mathrm{FH}]$. Let us fix some notations. We work on the manifold $X=S^{1} \times Y$. Denote by $D$ the group of all skew products $(t, y) \rightarrow(t+\alpha, h(t) y)$ where $\alpha \in S^{1}$ and $h: S^{1} \rightarrow G$ is a smooth loop. Let $D_{0}$ be its subgroup consisting of maps of the form $(t, y) \rightarrow(t, h(t) y)$. We write $S_{\alpha}$ for the shift $(t, y) \rightarrow(t+\alpha, y)$.

Consider the set of all mappings of the form $\phi^{-1} \circ S_{\alpha} \circ \phi$ where $\phi \in D_{0}$ and $\alpha \in S^{1}$. Its closure in $D$ can be identified in an obvious way with the set $\mathcal{R}$ introduced in 1.2, and in this appendix we use for it the same notation $\mathcal{R}$. 


\subsection{Strict ergodicity}

Consider a collection $\mathcal{C}$ of all subsets of $\mathcal{R}$ of the form

$$
C(F, \varepsilon)=\left\{T \in \mathcal{R} \mid \inf _{N \in \mathbf{N}}\left\|\frac{1}{N} \Sigma_{j=0}^{N-1} F \circ T^{j}\right\|<\varepsilon\right\},
$$

where $F$ runs over all continuous functions with zero mean on $X$, and $\varepsilon>0$. It is easy to see that the intersection of all sets from $\mathcal{C}$ consists of strictly ergodic diffeomorphisms. Thus for the existence of strictly ergodic skew products it suffices to show that the intersection of all sets from $\mathcal{C}$ is non-empty. Consider a countable subcollection $\mathcal{C}^{\prime}$ of $\mathcal{C}$ which consists of all sets of the form $C\left(F_{j}, \frac{1}{k}\right)$, where $\left\{F_{j}\right\}$ is a countable dense subset of the space of continuous functions with zero mean on $X$, and the number $k$ runs over the natural numbers. Obviously every set from $\mathcal{C}$ contains a set from $\mathcal{C}^{\prime}$, thus the intersection of all sets from $\mathcal{C}$ is equal to the intersection of all sets from $\mathcal{C}^{\prime}$.

Now the strategy is as follows. The collection $\mathcal{C}$ consists of subsets which are open in $\mathcal{R}$. Since the group $G$ is closed in $\operatorname{Diff}(Y)$, the set $\mathcal{R}$ (with the topology induced from $\operatorname{Diff}(X))$ has the Baire property. ${ }^{7}$ Therefore it suffices to show that the subsets from $\mathcal{C}$ are dense in $\mathcal{R}$.

Lemma 5.3.A. Let $C$ be a set from $\mathcal{C}$ and $r$ be a rational number. There exists $\phi \in D_{0}$ which commutes with $S_{r}$ and such that for every irrational number $\alpha$ the diffeomorphism $\phi^{-1} \circ S_{\alpha} \circ \phi$ belongs to $C$.

\subsection{The final argument}

Assume the Lemma. We claim that for every rational $r$ and every $C \in \mathcal{C}$ the shift $S_{r}$ belongs to the closure of $C$. Indeed, take $\phi$ from the Lemma and choose a sequence $\left\{\alpha_{j}\right\}$ of irrational numbers which converges to $r$. Then $\left\{\phi^{-1} \circ S_{\alpha_{j}} \circ \phi\right\}$ is a sequence of elements of $C$ which converges to $S_{r}$, and the claim follows. Since rational numbers are dense in the circle, we conclude that every (rational or irrational) shift is in the closure of $C$. Note that for every $\psi \in D_{0}$ and $C \in \mathcal{C}$ the set

$$
\left\{\psi \circ f \circ \psi^{-1} \mid f \in C\right\}
$$

is again contained in $\mathcal{C}$. Thus every element of the form $\psi^{-1} \circ S_{\alpha} \circ \psi$ with $\psi \in D_{0}$ belongs to the closure of $C$. Since by definition these elements are dense in $\mathcal{R}$, we get that $C$ is dense in $\mathcal{R}$. As it was explained in 5.3 this completes the proof of existence of smooth strictly ergodic skew products.

\footnotetext{
7 This is exactly the place where we use that $G$ is closed in $\operatorname{Diff}(Y)$. It seems, however, that one can prove the theorem without this assumption. For that purpose one should work with a different cleverly chosen topology on $G$.
} 


\subsection{Proof of 5.3.A}

Fix a set $C=C(F, \epsilon)$ from $\mathcal{C}$ and a rational number $r$. Given a loop $g: S^{1} \rightarrow G$ consider the integral

$$
I(y)=\int_{0}^{1} F\left(t, g(t)^{-1} y\right) d t .
$$

Using Property 2.1.A we can choose $g$ in such a way that $g(t+r)=g(t)$ for all $t$, and $|I(y)|<\varepsilon / 2$ for all $y$. Define an element $\phi \in D_{0}$ by $\phi(t, y)=$ $(t, g(t) y)$. It commutes with $S_{r}$. Take an irrational $\alpha$. Write the ergodic sum

$$
\frac{1}{N} \Sigma_{j=0}^{N-1} F \circ\left(\phi^{-1} \circ S_{\alpha} \circ \phi\right)^{j}
$$

as $F_{N} \circ \phi$, where

$$
F_{N}=\frac{1}{N} \Sigma_{j=0}^{N-1}\left(F \circ \phi^{-1}\right) \circ S_{\alpha}^{j} .
$$

Since the shift of the circle $t \rightarrow t+\alpha$ is strictly ergodic, and the family $\left\{F\left(t, g(t)^{-1} y\right)\right\}, y \in Y$ of functions $S^{1} \rightarrow \mathbf{R}$ is compact, the ergodic sum above converges uniformly to $I(y)$ when $N$ goes to infinity. In particular for large $N$ holds $\left\|F_{N} \circ \phi\right\|=\left\|F_{N}\right\|<\varepsilon$, and we conclude that $\phi$ is as needed. This completes the proof.

Acknowledgements. I thank Viktor L. Ginzburg, Eli Glasner, Dusa McDuff, Vitali Milman, Jürgen Moser, and Paul Seidel for very useful discussions, consultations and suggestions. I am very grateful to Felix Schlenk for pointing out a number of inaccuracies in the first version, and to the referee for valuable comments. This paper was written during my stay at ETH-Zürich. I thank the Forschungsinstitut für Mathematik for the hospitality.

\section{References}

[AK] D. Anosov, A. Katok: New examples in smooth Ergodic Theory. Ergodic diffeomorphisms. Trans. Moscow Math. Soc. 23, 1-35 (1970)

[AM] M. Abreu, D. McDuff: (in preparation)

[BB] D. Berend, V. Bergelson: Ergodic and mixing sequences of transformations. Ergodic Theory Dyn. Syst. 4, 353-366 (1984)

[BP] M. Bialy, L. Polterovich: Invariant tori and Symplectic Topology. Am. Math. Soc. Transl. (2) 171, 23-33 (1996)

[CFS] I. Cornfeld, S. Fomin, Ya. Sinai: Ergodic Theory. Springer 1982

[E1] L.H. Eliasson: Ergodic skew-systems on $T^{d} \times S O(3, \mathbf{R})$ (Preprint ETH Zurich, 1991)

[E2] L.H. Eliasson: Reducibility and point spectrum for linear quasi-periodic skew products. Proceedings of the ICM, Vol. II, pp. 779-787. Berlin, 1998

[F1] H. Furstenberg: Strict ergodicity and transformations of the torus. Am. J. Math. 83, 573-601 (1961)

[F2] H. Furstenberg: Recurrence in Ergodic Theory and Combinatorial Number Theory. Princeton University Press, Princeton, NJ 1981 
[FH] A. Fathi, M. Herman: Existence de diffeomorphismes minimaux. Asterisque 49, 37-59 (1977)

[GW] S. Glasner, B. Weiss: On the construction of minimal skew products. Israel J. Math. 34, 321-336 (1979)

$[\mathrm{H}] \quad$ H. Hofer: On the topological properties of symplectic maps. Proc. R. Soc. Edinb. Sect. A 115, 25-38 (1990)

[HZ] H. Hofer, E. Zehnder: Symplectic invariants and Hamiltonian dynamics. Birkhäuser, Basel 1994

[He] M. Herman: Construction de diffeomorphismes ergodiques. (Unpublished manuscript)

[K] A. Katok: Ergodic perturbations of degenerate integrable Hamiltonian systems. Izv. Akad. Nauk. SSSR Ser. Mat. 37, 539-576 (1973) (in Russian). English translation: Math. USSR Izvestija 7, 535-571 (1973)

[Ki] Y. Kifer: Random dynamics and its applications. Proceedings of the ICM, Vol. II, pp. 809-818. Berlin, 1998

[Kr] U. Krengel: Ergodic Theorems. Walter de Gruyter, Berlin, New York 1985

[LM] F. Lalonde, D. McDuff: Hofer's $L^{\infty}$-geometry: energy and stability of Hamiltonian flows I,II. Inv. Math. 122, 1-69 (1995)

[LMP] F. Lalonde, D. McDuff, L. Polterovich: On the Flux Conjectures. CRM Proc. Lect. Notes 15, 69-85 (1998)

[MS] D. McDuff, D. Salamon: Introduction to Symplectic Topology. Clarendon Press, Oxford 1995

[N] M. Nerurkar: On the construction of smooth ergodic skew-products. Ergodic Theory Dyn. Syst. 8, 311-326 (1988)

[P1] L. Polterovich: Hamiltonian loops and Arnold's principle. Am. Math. Soc. Transl. (2) 180, 181-187 (1997)

[P2] L. Polterovich: Precise measurements in Symplectic Topology. Proceedings of the 2-nd European Congress of Mathematics, Budapest, 1996. Vol. II, pp. 159-166. Birkhäuser, Basel 1998

[P3] L. Polterovich: Geometry on the group of Hamiltonian diffeomorphisms. Proceedings of the ICM, Vol. II, pp. 401-410. Berlin, 1998

[Si] J.-C. Sikorav: Systemes Hamiltoniens et topologie symplectique. ETS Editrice, Pisa 1990

[U] I. Ustilovsky: Conjugate points on geodesics of Hofer's metric. Diff. Geometry Appl. 6, 327-342 (1996) 